\title{
The role of monocyte to high-density lipoprotein cholesterol ratio in prediction of increased systemic inflammation and the risk of cardiovascular disease in endometriosis
}

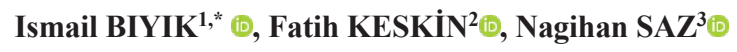 \\ ${ }^{1}$ Kütahya Health Sciences University, Kütahya, Turkey \\ ${ }^{2}$ Private Balkan Hospital, Edirne, Turkey \\ ${ }^{3}$ Private Kent Hospital, İzmir, Turkey
}

Received: $18.05 .2020 \quad \bullet \quad$ Accepted/Published Online: $05.11 .2020 \quad \bullet \quad$ Final Version: 14.03 .2021

\begin{abstract}
Endometriosis occurs in about 5-10 in 100 women of reproductive age. The pathophysiology of endometriosis is controversial. Some studies claimed an association between endometriosis and increased levels of inflammatory factors in peritoneal fluid and/or peripheral blood. Monocyte / HDL cholesterol ratio (MHR) and neutrophil/lymphocyte ratio (NLR) are inflammatory markers and are used as predictors and prognostic indicators of mortality and morbidity in many diseases. In this study, we aimed to investigate whether Monocyte / HDL cholesterol ratio (MHR) and neutrophil/lymphocyte ratio (NLR) are increased in endometriosis as in patients with chronic inflammation and cardiovascular diseases. This is a retrospective case-control study conducted with 87 women, 45 in the endometriosis group and 42 in the control group. The demographic data, biochemical, complete blood count parameters and lipid profile of the cases were recorded and compared between the groups. The mean age of the endometriosis group was 33.88 years and was older than the control group. In terms of other demographic data, there were no difference between the two groups. Although the platelet distribution width and triglyceride values of the endometriosis group were higher than controls, they were interpreted as clinically insignificant. There were no significant differences between the groups in terms of other laboratory parameters including MHR and NLR. In this study, MHR and NLR are found similar in endometriosis and control groups. Further studies are needed to investigate the relationship between increased systemic inflammation.
\end{abstract}

Keywords: endometriosis, monocyte/hdl cholesterol ratio, neutrophil/lymphocyte ratio, inflammation

\section{Introduction}

Endometriosis is a disease characterized by the presence of endometrial gland and stroma outside the uterus (Vercellini et al., 2014). Endometriotic implants are most frequently observed on peritoneal surfaces in the pelvis. Endometriosis is an estrogen dependent benign disease that occurs in \%5-10 women at reproductive age with the most common age range being 25-35 (Vercellini et al., 2014). Non-invasive marker does not exist to diagnose endometriosis (Coutinho et al., 2019). Transvaginal ultrasound and magnetic resonance are imaging methods to diagnose endometriosis non-invasively (Peiris et al., 2018).

Although it is one of the most common gynecological diseases in the reproductive age, the pathophysiology of endometriosis is controversial. There are studies linking endometriosis with increased levels of interleukin-1, interleukin-6, and tumor necrosis factor- $\alpha$ in peritoneal fluid (Bedaiwy et al., 2002) and peripheral blood (Koumantakis et al., 1994; Gupta et al., 2006). In addition to findings of increased oxidative stress in peritoneal fluid and peripheral blood, decreased antioxidant levels have also been reported (Verit et al., 2008).

Besides increased oxidative stress and chronic inflammation, endometriosis is also associated with atherogenic lipid profile (Mu et al., 2016). Increased LDL and decreased HDL levels were found in the blood of patients with endometriosis (Melo et al., 2010).

Nearly $3-8 \%$ of circulating leukocytes are monocytes and monocytes and they are the components of natural immune system. Monocytes transform into macrophages when they reach tissues. Macrophages play a central role in inflammation with phagocytosis, release of inflammatory cytokines and free oxygen radicals, activation of the acquired immune system (Auffray et al., 2009; Linker et al., 2009). Increased oxidative stress and inflammation provided by monocytes and macrophages have beneficial effects for the human body as 
well as potential harmful effects. Excessive monocyte activation causes atherosclerosis, arthritis and multiple sclerosis by activating the prothrombotic pathway through platelets and endothelial cells. In contrast, high-density lipoprotein (HDL) cholesterol neutralizes the oxidation and macrophage migration caused by low-density lipoprotein (LDL) cholesterol in monocytes (Parthasarathy et al., 1990). HDL cholesterol has anti-inflammatory properties by suppressing proliferation, differentiation and activation of monocyte progenitor cells (Westerterp et al., 2012).

Monocyte / HDL cholesterol ratio (MHR) is a recently identified inflammatory marker, a predictor and prognostic indicator of mortality and morbidity in many diseases (Canpolat et al., 2016). The advantage of MHR in showing chronic inflammation and increased cardiovascular risk is that it is cheap and easily applicable. MHR increases in diseases characterized by chronic inflammation. MHR is measured high in cardiovascular diseases (Cetin et al., 2016), hypertension (Aydin et al., 2017), metabolic syndrome (Vahit et al., 2017), polycystic ovary syndrome (Usta et al., 2018).

Leukocytes are immune system cells exist in peripheral blood. Leukocyte subtypes include cells such as neutrophils, lymphocytes, monocytes and eosinophils. There are many studies that compare leukocyte cell subtypes to each other and associate these rates with increased inflammatory status and oxidative stress. Peripheral hematological markers such as neutrophil-lymphocyte ratio (NLR) are used as an indicator of systemic inflammation (Zhang et al., 2020). Although some studies demonstrated that NLR is high in patients with endometriosis (Turgut et al., 2019), others believe that there is no difference when compared to controls (Yavuzcan et al., 2013). It is alleged that increased cardiovascular risk and chronic inflammation present in patients with endometriosis. However, it is not clear whether MHR is increased or not in endometriosis. In this study, we aimed to investigate whether Monocyte / HDL cholesterol ratio (MHR) and neutrophil/lymphocyte ratio (NLR) are increased in endometriosis as in patients with other chronic inflammatory disorders and cardiovascular diseases.

\section{Materials and method}

This is a retrospective case control study conducted in two community hospital (Bursa Karacabey and Edirne Sultan 1. Murat Hospitals) between January 2018 and October 2019. All of the medical records of patients (Laboratuary tests and ultrasonographic reports) aged between 18-45 years who presented to one of two community hospital during the study period were reviewed. The ethic approval was given by scientific research ethics committee of Trakya University Faculty of Medicine (TÜTF-BAEK 2019/395). This study was carried out in accordance with the Helsinki Declaration Principles. Sample size calculation was made by PASS 11 program; \%80 power, predicted ratio between groups is 1.40 , alpha significance level is taken as $0.05 n=64$ (in case group:
32, control group: 32). This study was conducted with 87 women, 45 in the endometriosis group and 42 in the control group.

Diagnosis of endometriosis was made based on ESHRE 2013 Guideline recommendations. All women who were eligible of these criteria were included in the study (Dunselman et al., 2014). Patients who were diagnosed histopathologically by laparoscopic examination or who had typical endometrioma appearance (such as ground glass appearance) on ultrasonography are accepted as endometriosis. Twenty-nine women had operation either by diagnostic laparoscopy or laparoscopic cystectomy. Remaining 16 women were diagnosed sonographically with typical endometrioma images based on symptoms such as dysmenorrhea, dysparenuria and infertility. These were the cases that operation was not indicated for these symptoms. None of the cases were on oral contraceptives, progesteron or GnRH analogs during sample collection. The control group composed of women with similar demographic characteristics with the study group but were not suspected or diagnosed with endometriosis were included. The control group composed of fertile women who attended with complaints unrelated to endometriosis and had no findings on ultrasonography suggesting endometriosis such as endometrioma. Blood samples were collected randomly irrespective of the cycle of cases. Those with active infections, inflammatory disease, autoimmune disease, cancer, dyslipidemia, cardiovascular disease, diabetes, hormone-releasing tumor; patients using dyslipidemia drug, oral contraceptive, dienogest, gonadotropin hormone releasing hormone analogue, antihypertensive, steroid, antidiabetic drug, anticoagulant and antiplatelet drug were excluded. Demographic data such as age, height, weight, body mass index, gravida, parity, number of children living, biochemical, complete blood count parameters and lipid profile were recorded. Glucose, ALT (alanine aminotransferase), AST (aspartate aminotransferase), urea, creatinine levels were performed with Beckman Coulter, Unicel DXI 600 (Access Immunoassay System). Complete blood count was studied with an automatic hematology analyzer (Cell-Dyn 3700 Abbott, Abbott Park, IL). HDL cholesterol, LDL cholesterol, total cholesterol and triglyceride levels from lipid profile measurements were performed with AutoAnalyzer Cobas Integra 800 (Roche Diagnostics GmbH; Mannheim, Germany).

\subsection{Statistical analysis}

Categorical variables were presented as numbers and percentages, while numerical variables were presented with mean, median and standard deviation. Monocyte / HDL cholesterol ratios were calculated in both case and control groups. When comparing all numerical data between the two groups, Student's T Test was used for the normal distribution. Chi-square or Fisher Exact Test was used to compare categorical variables. All statistical analysis SPSS 21 package program was used. 


\section{Results}

The study was conducted with 87 women, 45 in the endometriosis group and 42 in the control group. While the mean age of the endometriosis group was 33.88 years, the mean age of the control group was 28.78 years and the difference was statistically significant $(p=0.001)$. There were no differences between the two groups in terms of other demographic data $(\mathrm{p}>0.05)$ (Table 1).

Table 1. Comparison of demographic datas of groups

\begin{tabular}{|c|c|c|c|}
\hline Variable & Endometriosis & Control & P \\
\hline Age (year) & $33.88 \pm 6.82$ & $28.78 \pm 6.52$ & 0.001 \\
\hline Gravidity & $1(0-5)$ & $1(0-4)$ & 0.787 \\
\hline Parity & $1(0-3)$ & $1(0-3)$ & 0.870 \\
\hline Abortion & $0(0-3)$ & $0(0-2)$ & 0.514 \\
\hline Live child & $1(0-3)$ & $0.5(0-3)$ & 0.369 \\
\hline Weight $(\mathrm{kg})$ & $64.58 \pm 11.88$ & $62.71 \pm 10.30$ & 0.436 \\
\hline Height $(\mathrm{cm})$ & $163.42 \pm 5.56$ & $166.14 \pm 7.25$ & 0.052 \\
\hline BMI* $\left(\mathrm{kg} / \mathrm{m}^{2}\right)$ & $24.21 \pm 4.53$ & $22.70 \pm 3.23$ & 0.078 \\
\hline
\end{tabular}

Although the platelet distribution width and triglyceride values of the endometriosis group were higher, they were interpreted as clinically insignificant $(p=0.046$ and $p=0.049$, respectively). There were no significant differences between the groups when analysis was performed with the other laboratory parameters including MHR and NLR ( $p>0.05)$. (Table 2).

Table 2: Comparison of laboratory datas of groups

\begin{tabular}{|c|c|c|c|}
\hline Variable & Endometriosis & Control & $\mathbf{P}$ \\
\hline ALT (U/L) & $14.65 \pm 6.82$ & $13.21 \pm 6.52$ & 0.281 \\
\hline AST (U/L) & $16.55 \pm 5.58$ & $17.12 \pm 4.70$ & 0.619 \\
\hline Glucose (mg/dL) & $90.79 \pm 8.07$ & $89.14 \pm 6.75$ & 0.312 \\
\hline Urea $(\mathrm{mg} / \mathrm{dL})$ & $25.85 \pm 21.82$ & $21.92 \pm 5.56$ & 0.278 \\
\hline $\begin{array}{l}\text { Creatinin } \\
(\mathrm{mg} / \mathrm{dL})\end{array}$ & $0.65 \pm 0.14$ & $0.69 \pm 0.10$ & 0.170 \\
\hline $\begin{array}{l}\text { HDL cholesterol } \\
(\mathrm{mg} / \mathrm{dL})\end{array}$ & $62.88 \pm 22.58$ & $57.04 \pm 11.31$ & 0.135 \\
\hline $\begin{array}{l}\text { LDL cholesterol } \\
(\mathrm{mg} / \mathrm{dL})\end{array}$ & $104.71 \pm 33.51$ & $106.95 \pm 21.57$ & 0.710 \\
\hline $\begin{array}{l}\text { Total cholesterol } \\
(\mathrm{mg} / \mathrm{dL})\end{array}$ & $182.63 \pm 36.28$ & $173.09 \pm 25.45$ & 0.162 \\
\hline $\begin{array}{l}\text { Triglycerides } \\
(\mathrm{mg} / \mathrm{dL})\end{array}$ & $117.22 \pm 51.21$ & $91.84 \pm 49.41$ & 0.049 \\
\hline $\begin{array}{l}\text { Hemoglobin } \\
(\mathrm{g} / \mathrm{dL})\end{array}$ & $12.48 \pm 1.41$ & $12.79 \pm 0.92$ & 0.230 \\
\hline Hematocrite (\%) & $37.58 \pm 3.65$ & $38.45 \pm 2.34$ & 0.191 \\
\hline Platelet $\left(10^{3} / \mu \mathrm{l}\right)$ & $275.81 \pm 65.87$ & $268.90 \pm 50.64$ & 0.547 \\
\hline $\operatorname{MCV}(\mathrm{fl})$ & $83.58 \pm 6.61$ & $85.43 \pm 5.72$ & 0.172 \\
\hline MPV (fl) & $9.39 \pm 1.68$ & $9.48 \pm 1.15$ & 0.779 \\
\hline Platelectrict (\%) & $0.25 \pm 0.05$ & $0.25 \pm 0.04$ & 0.924 \\
\hline PDW (\%) & $16.04 \pm 0.41$ & $15.88 \pm 0.32$ & 0.046 \\
\hline WBC $\left(10^{3} / \mu \mathrm{l}\right)$ & $\begin{array}{l}7427.95 \pm 2007 . \\
18\end{array}$ & $\begin{array}{l}6882.85 \pm 1335 . \\
57\end{array}$ & 0.144 \\
\hline $\begin{array}{l}\text { Monocyte } \\
\left(10^{3} / \mu \mathrm{l}\right)\end{array}$ & $0.68 \pm 1.23$ & $0.97 \pm 3.48$ & 0.598 \\
\hline $\begin{array}{l}\text { Neutrophile } \\
\left(10^{3} / \mu \mathrm{l}\right)\end{array}$ & $4.42 \pm 1.44$ & $3.98 \pm 1.07$ & 0.114 \\
\hline $\begin{array}{l}\text { Lymphocyte } \\
\left(10^{3} / \mu \mathrm{l}\right)\end{array}$ & $2.42 \pm 0.76$ & $2.25 \pm 0.46$ & 0.211 \\
\hline MHR & $0.0095 \pm 0.0065$ & $0.0181 \pm 0.0658$ & 0.391 \\
\hline NLR & $1.93 \pm 0.78$ & $1.84 \pm 0.65$ & 0.567 \\
\hline
\end{tabular}

\section{Discussion}

In this study, HMR and NLR ratios which increases in systemic inflammation that are associated with increased risk of cardiovascular disease in the peripheral circulation were found similar between endometriosis and control groups. Although the platelet distribution width (PDW) and triglyceride values of the endometriosis group were higher, they were interpreted as clinically insignificant $(\mathrm{p}=0.046$ and $\mathrm{p}=0.049$, respectively). An increased PDW value has been found in various cardiovascular diseases (Li et al., 2020). Some studies reported that triglyceride-rich lipoproteins as a causal factor for cardiovascular disease (Toth, 2016). In our study, other inflammatory markers except TG and PDW were found similar in both groups. With the data of our study, we think that women with endometriosis do not have increased systemic inflammatory status and cardiovascular risk.

Endometriosis is an estrogen-dependent, progressive disease. Inflammatory response, genetic and environmental factors, hormonal regulation play important role in its pathogenesis (Khan et al., 2008). Endometriosis is considered to activate the pelvic inflammatory process, and the functions of immune-related cells in the peritoneal cavity change.

Previously published studies have found that inflammatory markers increase in the peritoneal cavity of patients with endometriosis (Barrier, 2010). Some studies report that urocortin, highly sensitive c reactive protein (CRP), interleukin 2 (IL-2), interleukin 4 (IL-4), interleukin 10 (IL10 ), interferon gamma (IFN- $\gamma$ ) and serum amyloid A levels in the systemic circulation are increased due to increased inflammatory response in endometriosis (Abrao et al., 1997). Ectopic endometrium tissue has been shown to secrete interleukin 6 in patients with endometriosis (Akoum et al., 1996). It has even alleged to be an independent marker for predicting endometriosis. Intercellular adhesion molecule 1 (ICAM-1) increases in peritoneal fluid of endometriosis cases and regulates natural killer activity (Daniel et al., 2000). Additionally, some studies showed that the levels of soluble ICAM-1 increase in serum of patient with endometriosis $(\mathrm{Wu}$ et al., 1998), while others found similar levels to the control group (De Placido et al., 1998).

Systemic inflammation parameters may not increase despite local inflammation in the peritoneal cavity. Bedaiwy et al. demonstrated that IL-2 levels were similar in the serum of endometriosis and controls (Bedaiwy et al., 2002). Myeloperoxidase (MPO) is a proinflammatory enzyme and neutrophil activation is a marker of oxidative stress. Dorien et al. demonstrated in their study that total and active MPO levels in the blood of endometriosis and control cases were similar (O et al., 2018). Thubert et al. found similar highly sensitive CRP levels in endometriosis and control groups (Thubert et al., 2014). In our study, the rate of NLR was similar in endometriosis and control cases similar to the study of Yavuzcan et al (Yavuzcan et al., 2013). In addition to their 
study, we also examined the rate of MHR. MHR rate was similar in endometriosis and control cases. We also claim that endometriosis is associated with increased local inflammation in the peritoneum, and not systemic inflammation.

Some newer studies describe that endometriosis is associated with comorbidities such as autoimmune disease, allergies, cancers and cardiovascular disease (Teng et al., 2016). Endometriosis is claimed to be associated with increased systemic inflammation, atherosclerosis, coronary artery disease and consequently increased cardiovascular morbidity, mortality (Wilson et al., 2011). Oxidative stress and abnormal lipid profile are risk factors for cardiovascular disease and there are studies showing that they are also abnormal in cases of endometriosis (Dhalla et al., 2000). Increased reactive oxygen radicals, oxidized LDL and antioxidant expression have been shown to be altered in patients with endometriosis (Gupta et al., 2006). Endometriosis has also been correlated with atherogenic lipid profile, such as increased LDL, non-HDL lipoprotein, and decreased HDL levels (Melo et al., 2010). In a large case series study, it has been shown that the risk of developing coronary heart disease increases in patients with laparoscopically confirmed endometriosis ( $\mathrm{Mu}$ et al., 2016). In this study, we claim that there is no increased risk of cardiovascular disease in patients with endometriosis because of the similar MHR rate in endometriosis and control cases. There is strong evidence about the changes in cell and cytokine profile representing local inflammation in the peritoneal fluid in patients with endometriosis. However, the issue of whether systemic inflammation has increased is controversial.

In this study, HMR and NLR ratios associated increased systemic inflammation and increased risk of cardiovascular disease, were found similar in endometriosis and control cases. Our findings support the view that endometriosis is a local inflammatory pelvic disease rather than a systemic one. Further large-scale studies are needed to investigate the relationship between increased systemic inflammation and the risk of cardiovascular disease in endometriosis.

\section{References}

1. Abrao, M.S., Podgaec, S., Filho, B.M., Ramos, LO., Pinotti, JA., de Oliveira, R.M., 1997. The use of biochemical markers in the diagnosis of pelvic endometriosis. Hum Reprod. 12, 2523- 2527.

2. Akoum, A., Lemay, A., Paradis, I., 1996. Secretion of interleukin6 by human endometriotic cells and regulation by proinflammatory cytokines and sex steroids. Hum Reprod. 11, 2269- 2275.

3. Auffray, C., Sieweke, M.H., Geissmann, F., 2009. Blood monocytes: development, heterogeneity, and relationship with dendritic cells. Annu Rev Immunol. 27, 669-92.

4. Aydin, E, Ates, I., Fettah Arikan, M., Yilmaz, N., Dede, F., 2017. The ratio of monocyte frequency to HDL cholesterol level as a predictor of asymptomatic organ damage in patients with primary hypertension. Hypertens Res. 40, 758-64.

5. Barrier, B.F, 2010. Immunology of endometriosis. Clin Obstet
Gynecol. 53, 397-402.

6. Bedaiwy, M.A., Falcone, T., Sharma, R.K., Goldberg, J.M., Attaran, M., Nelson, D.R, Agarwal, A., 2002. Prediction of endometriosis with serum and peritoneal fluid markers: a prospective controlled trial. Hum Reprod. 17, 426-431.

7. Canpolat, U., Çetin, E.H., Cetin, S., Aydin, S., Akboga, M.K., Yayla, C., Turak, O., Aras, D., Aydogdu S., 2016. Association of Monocyte-to-HDL cholesterol ratio with slow coronary flow is linked to systemic inflammation. Clin Appl Thromb. 22, 476-82.

8. Cetin, M.S., Ozcan Cetin, E.H., Kalender, E., Aydin, S., Topaloglu, S., Kisacik, HL., Temizhan, A., 2016. Monocyte to HDL cholesterol ratio predicts coronary artery disease severity and future major cardiovascular adverse events in acute coronary syndrome. Heart Lung Circ. 25, 1077-86.

9. Coutinho, LM., Ferreira, M.C., Rocha, A.L.L., Carneiro, M.M., Reis, F.M., 2019. New biomarkers in endometriosis. Adv Clin Chem. 89, 59-77.

10. Daniel, Y., Geva, E., Amit, A., Eshed-Englender, T., Baram, A., Fait, G., Lessing, J.B., 2000. Do soluble cell adhesion molecules play a role in endometriosis? Am J Reprod Immunol. 2000, 160166.

11. De Placido, G., Alviggi, C., Di Palma, G., Carravetta, C., Matarese, G., Landino, G., Racioopi L., 1998. Serum concentrations of soluble human leukocyte class I antigens and of the soluble intercellular adhesion molecule-1 in endometriosis: relationship with stage and non-pigmented peritoneal lesions. Hum Reprod. 13, 3206-3210.

12. Dhalla, N.S., Temsah, R.M., Netticadan, T., Role of oxidative stress in cardiovascular diseases. J Hypertens. 18, 655-673

13. Dunselman, G.A., Vermeulen, N., Becker, C., Calhaz-Jorge, C., D'Hooghe, T., De Bie, B., Heikinheimo, O., Horne, A.W., Kiesel, L., Nap, A., Prentice, A., Saridogan, E., Soriano, D., Nelen, W., 2014. European Society of Human Reproduction and Embryology. ESHRE guideline: management of women with endometriosis. Hum Reprod. 29, 400-12.

14. Gupta, S., Agarwal, A., Krajcir, N., Alvarez, J.G., 2006. Role of oxidative stress in endometriosis. Reprod Biomed Online. 13, 126-134.;

15. Khan, K.N., Kitajima, M., Hiraki, H., Fujishita, A., Sekine, I., Ishimaru, T., Masuzaki, H., 2008. Immunopathogenesis of pelvic endometriosis: role of hepatocyte growth factor, macrophages and ovarian steroids. Am J Reprod Immunol. 60, 383-404.

16. Koumantakis, E., Matalliotakis, I., Neonaki, M., Froudarakis, G., Georgoulias, V., 1994. Soluble serum interleukin-2 receptor, interleukin-6 and interleukin- 1a in patients with endometriosis and in controls. Arch Gynecol Obstet. 255, 107-112.

17. Li G., Zhang Y., Zhu Z., Du J., 2020. Evaluation of platelet distribution width in hypertension with hyperhomocysteinemia. Clin Exp Hypertens. 42, 61-66.

18. Linker, R., Gold, R., Luhder, F., 2009. Function of neurotrophic factors beyond the nervous system: inflammation and autoimmune demyelination. Crit Rev Immunol. 29, 43-68.

19. Melo, A.S., Rosa-e-Silva, J.C., Rosa-e-Silva, A.C., Poli-Neto, O.B., Ferriani, R.A., Vieira, C.S., 2010. Unfavorable lipid profile in women with endometriosis. Fertil Steril. 93, 2433-2436.

20. Mu, F., Rich-Edwards, J., Rimm, E.B., Spiegelman, D., Missmer, S.A., 2016. Endometriosis and risk of coronary heart disease. Circ Cardiovasc Qual Outcomes. 9, 257-264

21. O, D.F., Waelkens, E., Peterse, D.P., Lebovic, D., Meuleman, C., Tomassetti, C., Peeraer K, Alvarez Real, A., Bosseloir, A., D'Hooghe, T., Fassbender, A., 2018. Evaluation of Total, Active, 
and Specific Myeloperoxidase Levels in Women with and without Endometriosis. Gynecol Obstet Invest. 83, 133-139.

22. Parthasarathy, S., Barnett, J., Fong, L.G., 1990. High-density lipoprotein inhibits the oxidative modification of low-density lipoprotein. Biochim Biophys Acta. 1044, 275-83.

23. Peiris, A.N., Chaljub, E., Medlock, D., 2018. Endometriosis. JAMA. 25, 320,2608.

24. Teng, S.W., Horng, H.C., Ho, C.H., Yen, M.S., Chao, H.T., Wang, P.H., 2016. Taiwan Association of Gynecology Systematic Review Group. Women with endometriosis have higher comorbidities: analysis of do mestic data in Taiwan. J Chin Med Assoc. 79, 577-582

25. hubert, T., Santulli, P., Marcellin, L., Menard, S., M'Baye, M., Streuli, I., Borghese, B., de Ziegler, D., Chapron, C., 2014. Measurement of hs-CRP is irrelevant to diagnose and stage endometriosis: prospective study of 834 patients. Am J Obstet Gynecol. 210, 533.e1-533.e10.

26. Toth PP., 2016. Triglyceride-rich lipoproteins as a causal factor for cardiovascular disease. Vasc Health Risk Manag. 12,171-183.

27. Turgut. A., Hocaoglu, M., Ozdamar, O., Usta, A., Gunay, T., Akdeniz, E., 2019. Could hematologic parameters be useful biomarkers for the diagnosis of endometriosis? Bratisl Lek Listy. $120,912-918$

28. Usta, A., Avci, E., Bulbul, C.B., Kadi, H., Adali, E., 2018. The monocyte counts to HDL cholesterol ratio in obese and lean patients with polycystic ovary syndrome. Reprod Biol Endocrinol. 10;16, 34 .

29. Vahit, D., Mehmet, K.A., Samet, Y., Hüseyin, E., 2017. Assessment of monocyte to high density lipoprotein cholesterol ratio and lymphocyte-to-monocyte ratio in patients with metabolic syndrome. Biomark Med. 11, 535-40.

30. Vercellini, P., Vigano, P., Somigliana, E., Fedele, L., 2014. Endometriosis: pathogenesis and treatment. Nat Rev Endocrinol. $10,261-275$.

31. Verit, F.F., Erel, O., Celik, N., 2008. Serum paraoxonase-1 activity in women with endometriosis and its relationship with the stage of the disease. Hum Reprod. 23, 100-104.

32. Westerterp, M., Gourion-Arsiquaud, S., Murphy, A.J., Shih, A., Cremers, S., Levine, R.L., Tall, A.R., Yvan-Charvet, L., 2012. Regulation of hematopoietic stem and progenitor cell mobilization by cholesterol efflux pathways. Cell Stem Cell. 11, 195-206.

33. Wilson, P.W., 2018 Evidence of systemic inflammation and estimation of coronary artery disease risk: a population perspective. Am J Med 2008;121(10, Suppl 1):S15-S20

34. Wu, MH., Yang, B.C., Hsu, C.C., Lee, Y.C., Huang, K.E., 1998. The expression of soluble intercellular adhesion molecule-1 in endometriosis. Fertil Steril. 70, 1139-1142.

35. Yavuzcan, A., Caglar, M., Ustun, Y., Dilbaz, S., Ozdemir, I., Yildiz, E., Ozkara, A., Kumru, S., 2013. Evaluation of mean platelet volume, neutrophil/lymphocyte ratio and platelet/lymphocyte ratio in advanced stage endometriosis with endometrioma. J Turk Ger Gynecol Assoc. 1; 14, 210-5.

36. Zhang, E., Gao, M., Gao, J., Xiao, J., Li, X., Zhao, H., Wang, J., Zhang, N., Wang, S., Liu, Y., 2020. Inflammatory and Hematological Indices as Simple, Practical Severity Predictors of Microdysfunction Following Coronary Intervention: A Systematic Review and Meta-Analysis. Angiology. 4, 3319719896472 . 